OPEN D AccEss Freely available online
www.banglajol.info/index.php/JSF
Journal of Science Foundation
July 2019, Vol. 17, No. $2, p p .67-70$
ISSN (Print) 1728-7855
DOI: https://doi.org/ 10.3329/jsf.v17i2.51143

Case Report

\title{
Schwanomma of the Left Iliohypogastric Nerve: A Case Report and Technical Consideration
}

\author{
Md. Shafiul Alam ${ }^{1}$, Kaisar Haroon ${ }^{2}$, Tayseer Farzana ${ }^{3}$, Forhad Ahmed ${ }^{4}$, Tania Taher $^{5}$
}

\begin{abstract}
Schwannomas are encapsulated tumors arising from Schwann cells of the nerve sheath, and are usually solitary sporadic lesions. The schwannoma of the ilio-hypogastric nerve especially in the retropetoneal space is very rare. We are reporting a case of the schwannoma of the left ilio-hypagastric nerve over the psoas muscle. The diagnosis was done by ultarsonogrsphy and MRI. The histopathological study confirmed the diagnosis. The tumour was excised through retro-peritoneal approach. By this approach total excision of the tumour with preservation of ilio-hypogastric nerve is possible. [Journal of Science Foundation, July 2019;17(2):67-70]
\end{abstract}

Keywords: Ilio-hypogastric nerve; Schwanomma; retroperitoneal approach; resection

[Reviewed: 3 April 2019; Accepted on: 1 June 2019; Published on: 1 July 2019]

\section{Introduction}

Schwannomas are encapsulated tumors arising from Schwann cells of the nerve sheath, and are usually solitary sporadic lesions ${ }^{1}$. Schwannomas are rarely found in the retroperitoneum, accounting for only $0.7 \%$ cases to $2.7 \%$ cases of all primary schwannomas (Fu et al., 2008). They can be found in any nerve trunk, except for cranial nerves I and II, and their usual location is the head, neck, the flexor surfaces of the extremities and the posterior mediastinum or the retroperitoneum (Theodosopoulos et al., 2008). The first peripheral nerve tumor to satisfy the currently acceptable clinical and histological criteria of this tumor was described in 1910 by Verocay, who gave it the name of neurinoma. The more descriptive terms of neurilemoma or neurilemoma were introduced by Stout (Claes et al., 1987).

\footnotetext{
${ }^{1}$ Associate Professor, Department of Neurosurgery, National Institute of Neurosciences \& Hospital, Dhaka, Bangladesh

${ }^{2}$ Assistant Professor, Department of Neurosurgery, National Institute of Neurosciences \& Hospital, Dhaka, Bangladesh

${ }^{3}$ Consultant, Department of Radiology \& Imaging, Popular Diagnostic Center, Mirpur, Dhaka, Bangladesh

${ }^{4}$ Medical Officer, Department of Neurosurgery, National Institute of Neurosciences \& Hospital, Dhaka, Bangladesh

${ }^{5}$ Assistant Professor, Department of Paediatrics, Holy Family Red Crescent Medical College Hospital, Dhaka, Bangladesh
}

Correspondence: Dr. Md. Shafiul Alam, Associate Professor, Department of Gamma Knife Neurosurgery, National Institute of Neurosciences \& Hospital, Sher-E-Bangla Nagar, Agargaon, Dhaka, Bangladesh; Email: dr_chapal@hotmail.com; Cell no.: $+8801711567358$

Copyright: (02019 Alam et al. Published by Journal of Science Foundation. This article is distributed under the terms of the Creative Commons Attribution 4.0 International CC BY-NC License (https://creativecommons.org/licenses/by-nc/4.0/). This license permits unrestricted use, distribution and reproduction in any medium, provided the original work is properly cited, you give appropriate credit to the original author $(s)$ and is not used for commercial purposes 
Schwannoma usually arises between the third and sixth decades of life, with an equal predilection for men and women (Tortorelli et al., 2007). Sachwannoma is a benign tumour. Malignancy in a solitary schwannoma is rare (Kuyumcuoglu and Germiyanoglu 1990). When tumours are slow-growing benign tumour with favourable prognosis, they are called ancient schwannoma. These lesions begin as solid tumours but as they become larger, they undergo spontaneous degeneration with areas of haemorrhage and cystic changes (Loke et al., 1998). The tumor is classically described in two forms. The fascicular type (Antoni A) is composed of compact spindle-shaped cells that are arranged in short bundles or interlacing fascicles. There may be nuclear palisading and formation of Verocay bodies. Areas of fibrosis, necrosis, and hemorrhage may occur with thrombosis of the blood vessels. The reticular type (Antoni B) is highly vascularized and shows a loose arrangement of the Schwann cells in an open network of cysts and reticular fibers. There is no palisading of nuclei. In some tumors both forms can exist (D'Silva et al., 2003) ${ }^{7}$. We are reporting a case of the schwannoma of the left ilio-hypagastric nerve over the psoas muscle. The diagnosis was done by ultarsonogrsphy and MRI.

\section{Case Presentation}

A 36-year-old female patient was presented to us with history of pain in the left lower limb for six months. She also complained for tingling and numbness in the left lower limb for the same duration and mild weakness of left lower limb for 15 days. The pain was gradually increasing and also radiated to left loin. On examination her left lower limb muscle power was $4 / 5$ and mild sensory impairment. There was no tenderness in left hip joint and Kop psoas test was negative. The ultrasonography of whole abdomen showed a well circumscribed homogenous lesion with cystic changes over the left psoas muscle along the length of left ilio-hypogastric nerve. The MRI of the Lumbosacral spine showed a round iso-intense lesion in the left psoas muscle which was brilliantly hyper intense after contrast uptake (Figure I). We had operated the patient under general anesthesia in right lateral position. We had approached the tumour through left retroperitoneal plane and had found a round shaped, capsulated tumour along the distribution of iliohypogastric nerve which was excised (Figure IIa \& IIb). The excised tumour was firm, yellowish, capsulated and well circumscribed. It was attached with a nerve which had come out through the L2 intervertebral foramen. The specimen was sent for histopathological study which revealed a benign schwannoma (Figure III). By this approach total excision of the tumour with preservation of ilio-hypogastric nerve is possible.

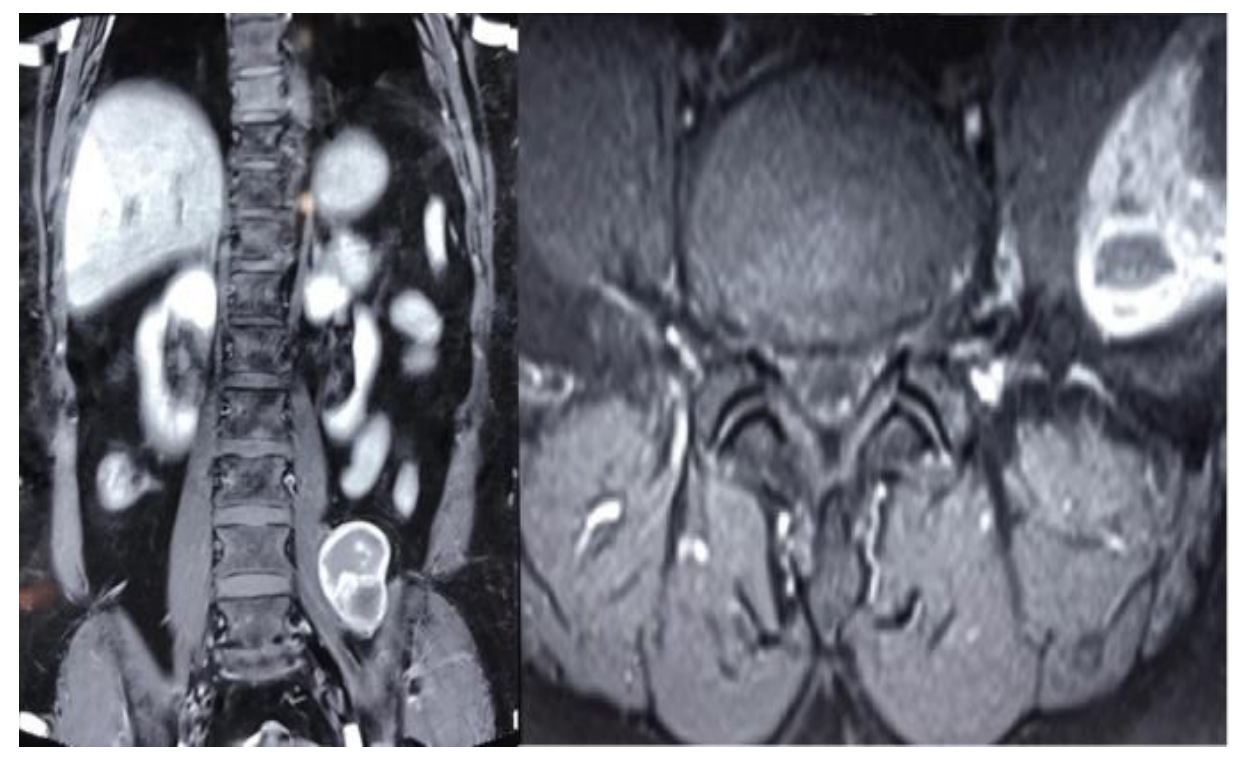

Figure I: T1 contrast MRI Coronal and axial section at the level of L4/L5 showing the tumour in the left psoas muscle 

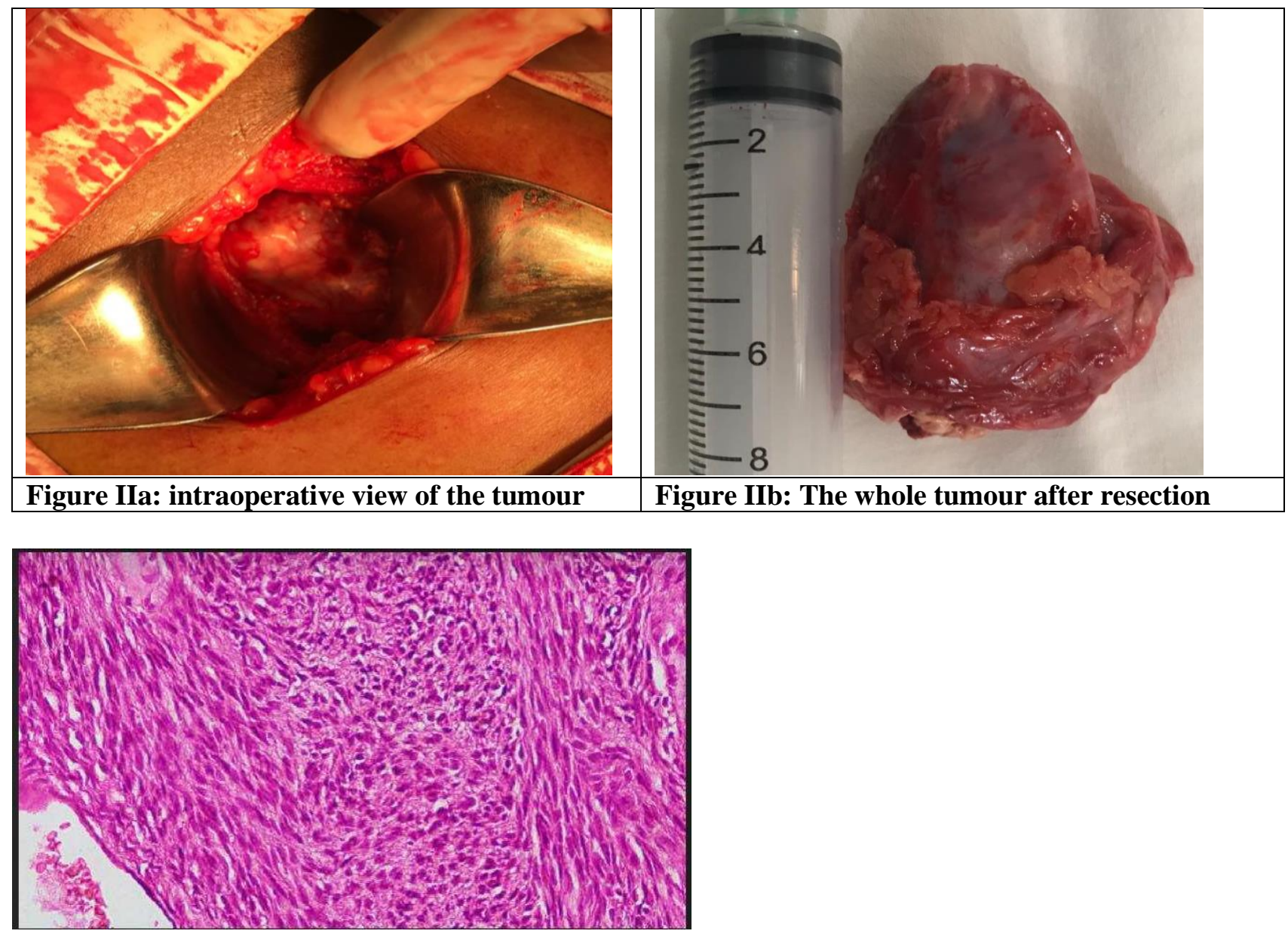

Figure III: Histopathological photomicrograph $(H \& E$ stain $)$

\section{Discussion}

Retroperitoneal schwannomas can be excised by retroperitoneal approach. Though it is also removed by use of laparoscopic approach (Seo et al., 2011). It is very convenient but there may be difficulties if the tumour was attached to the major vessels (Seo et al., 2011). Preoperative establishment of diagnosis is difficult in case of retroperitoneal schwannomas, however close relationship of retroperitoneal tumors with adjacent neural structures in imaging studies should raise a suspicion. Complete surgical resection is the treatment of choice. Histology and Immunohistochemistry confirms the diagnosis (Theodosopoulos et al., 2008). In our patient, during surgery the tumour had come from the left iliohypogastric nerve and the histopathological examination had confirmed it as schwannoma.

The retroperitoneum is flexible and nonrestrictive; therefore, a large, deeply situated tumor is usually present before patients have any symptoms. These masses tend to displace retroperitoneal structures such as the kidney and ureter but generally do not invade them (Daneshmand et al., 2003). In one series the size varied from 8.8 to $20 \mathrm{~cm}$ in the greatest diameter (Daneshmand et al., 2003). Our tumour was also about six cm in largest diameter.

Retroperotoneal schwannoma can present with varied symptoms. They mimic as a psoas abscess10, ureteric obstruction (Daneshmand et al., 2003), vague abdominal pain (Makhoul et al., 2017) or neurological problems as in our patient. Almost all Schwannoma show intense immunohistochemical staining for S-100 protein, confirming the neuroectodermal origin of the tumor cells (Tortorelli et al., 2007).

Furthermore, because even in benign Schwannoma a local recurrence rate of 10 to $20.0 \%$ cases has been described, probably due to incomplete surgical excision, a more conservative approach such as enucleation is not sufficient (Tortorelli et al., 2007). Therefore, complete excision is the surgery of choice. In our patient 
we had excised the tumour along with part of the healthy nerves on either side. The retroperitoneal approach affords direct access to lumbar paraspinal lesions. There are two other possible approaches. One is a transperitoneal approach via the midline, and the other a posterolateral approach through the sacrospinalis muscle. The former carries the risk of damaging the inferior vena cava and ureter. The latter has the disadvantage of severe postoperative muscle pain which may persist long after surgery (Hida et al., 1993). Therefore we had chosen the retroperitoneal approach. The approach was straight forward and the healing was satisfactory.

\section{Conclusion}

Psoas schwannoma is a rare tumour. It may present with different presentations. Abdominal ultrasonogram can determine its presence but it can be confirmed by abdominal MRI. Retroperitoneal approach and total excision can be carried out with no or minimum morbidity.

\section{References}

Claes H, Oyen R, Stessens R, Vereecken R. Solitary benign schwannoma in the psoas muscle. The journal of Urology. 1987;137(4):753-6

Daneshmand S, Youssefzadeh D, Chamie K, Boswell W, Wu N, Stein JP, et al. Benign retroperitoneal schwannoma: a case series and review of the literature. Urology. 2003;62(6):993-7

D'Silva KJ, Dwivedi AJ, Barnwell JM. Case report: Schwannoma of the psoas major muscle presenting with abdominal and back pain. Digestive diseases and sciences. 2003;48(8):1619

Fu CY, Lin CH, Yu TC, Lu TC, Hsieh CB. Solitary benign schwannoma of the psoas muscle: A case report. Visceral Medicine. 2008;24(3):249-51

Hida K, Iwasaki Y, Abe H, Itamoto K, Kaneda K. Schwannoma in the psoas muscle removed by the retroperitoneal approach. British Journal of Neurosurgery. 1993;7(2):213-5

Kuyumcuoglu U, Germiyanoglu C. Solitary benign schwannoma in the psoas muscle. International urology and nephrology. 1990;22(2):107-11

Liu Y-W, Chiu H-H, Huang C-C, Tu C-A. Retroperitoneal schwannoma mimicking a psoas abscess. Clinical Gastroenterology and Hepatology. 2007;5(9):A32

Loke T, Yuen N, Lo K, Lo J, Chan J. Retroperitoneal ancient schwannoma: Review of clinico-radiological features. Australasian Radiology. 1998;42(2):136-8

Makhoul E, Kamel R, Hanna N. Schwannoma of the psoas: An unusual cause of abdominal pain. Arab journal of gastroenterology. 2017;18(1):44-6

Seo IY, Boldbaatr Y, Choi KH. Laparoscopic Resection of Ancient Schwannoma Embedded in the Psoas Muscle. Surgical Laparoscopy Endoscopy \& Percutaneous Techniques. 2011;21(6):e336-e8

Theodosopoulos T, Stafyla VK, Tsiantoula P, Yiallourou A, Marinis A, Kondi-Pafitis A, et al. Special problems encountering surgical management of large retroperitoneal schwannomas. World Journal of Surgical Oncology. 2008;6(1):107

Tortorelli AP, Rosa F, Papa V, Rotondi F, Sanchez AM, Bossola M, et al. Retroperitoneal schwannomas: diagnostic and therapeutic implications. Tumori Journal. 2007;93(3):312-5 